\title{
Scienziati e confini culturali. Termalismo in Savoia nell'opera di Joseph Daquin (1732-1815)
}

Dino Carpanetto

\section{Summary}

The essay sets out to explain the general orientation of the studies, the problems, the perspectives and the research that were done by Joseph Daquin (1732-1815), a physician born in Chambéry (Savoy, Kingdom of Sardinia). He had a secular education at the Faculty of Medicine of Turin, where he earned his degree under Ignazio Somis and Vitaliano Donati. The aim of this essay is to give a critical contribution to cultural history and examine the origins of thermalism with particular emphasis on therapy, physics experiments, and its relation to political and social structure. Daquin was a witness of the advancement of science beyond the boundaries of scientific Enlightenment. His overriding intellectual concern was with the meaning and impact of chemistry theories on medical practices. The author has published an interesting Analyse des eaux thermales d'Aix en Savoie (1773), a natural experimental history of mineral waters in Aix-les-Bains.

Keywords: thermalism; scientific enlightenment; Savoy; Turin; Chambéry

\section{Riassunto}

Il saggio mira a fornire un orientamento generale sugli studi, i problemi, le prospettive e le ricerche effettuate da Joseph Daquin (1732-1815), medico, originario di Chambéry (Savoia, Regno di Saredegna). Egli compì il suo corso

* Ringrazio Cinzia Rando per il prezioso aiuto che mi ha prestato nella preparazione di questo articolo.

Prof. Dino Carpanetto, Università di Torino, Dipartimento di Storia, Via Sant'Ottavio 20, I-10124 Torino (dino.carpanetto@unito.it). 
di studi universitari alla Facoltà di Medicina di Torino, dove si laureò sotto la guida di Ignazio Somis e Vitaliano Donati. Obiettivo dell'articolo è di fornire un contributo critico alla storia culturale, esaminando le origini degli studi sul termalismo e sottolineando in particolare quelli sulla terapia, gli esperimenti di fisica e le relazioni di tali indagini con la struttura politica e sociale. Daquin fu un testimone dell'avanzamento delle scienze in un'area di frontiera dell'Illuminismo scientifico. Il peculiare interesse dei suoi studi risiede nel significato e nell'impatto delle teorie chimiche con le pratiche della medicina. Fu autore di un'interessante Analyse des eaux thermales d'Aix en Savoie (1773), una storia naturale e sperimentale delle acque minerali di Aix-les-Bains.

Il tema degli scambi culturali tra Piemonte e Savoia nella tarda età moderna richiama all'attenzione un approccio ormai classico nella storia delle idee, fornito come è di una ricca tradizione di studi che vanno dalla Crisi della coscienza europea di Paul Hazard del 1935 al Settecento riformatore (19691990) di Franco Venturi. Secondo tale metodo la circolazione di idee e di uomini, di cose e di esperienze, sia nello spazio, da un territorio culturale ad un altro, sia nel tempo, dal passato al presente, opera come una potente leva di modernizzazione che fa perno sul mondo dei libri, delle accademie, della massoneria, degli insegnamenti derivanti dai viaggi, con tutte le contaminazioni a cui danno luogo i processi di comunicazione. Tuttavia tale modello non può essere accolto in una versione semplicistica, nella quale la mobilità sia di per sé considerata un motore di accelerazione del cambiamento, in un mondo che diventa aperto, cosmopolita, capace di accogliere e integrare le diversità. In altri termini, il tema della circolazione va analizzato, come ricorda Daniel Roche, in rapporto al contesto di accoglienza, alle diversità nazionali e regionali, religiose ed economiche che frammentano il mondo occidentale, perché «la circolazione veicola anche i luoghi comuni e i pregiudizi, le rappresentazioni grossolane, l'incomprensione, persino il rifiuto dell'altro» ${ }^{1}$. E inoltre non bisogna dimenticare che nell'età moderna la mobilità era limitata e l'orizzonte dell'esperienza umana per lo più chiuso e ristretto, così che l'identità del luogo appariva in grado di condizionare le coordinate morali e materiali in cui si svolgeva la vita della maggioranza delle persone.

1 Roche 2007, 129. 
Alla luce di quanto detto occorre considerare le specifiche relazioni di scambio tra due spazi - il Piemonte e la Savoia - che appartenevano ad un unico stato. Per tale motivo potrebbero essere studiati come poli di un unico sistema complessivo, facendo valere piuttosto le chiavi interpretative basate sul nesso centro-periferia. Tale nesso tuttavia non dà pienamente conto delle specificità e delle connessioni. Basti pensare al fatto che si era costruito un rapporto istituzionale tra Torino e Chambéry in cui gli organismi della giustizia che davano vita ai rispettivi Senati erano separati. Le stesse élites si organizzavano in funzione sia delle cariche ricoperte nei rispettivi ambiti regionali sia dei ruoli di servizio nello stato, e al tempo stesso coltivavano posizioni di comando nelle strutture ecclesiastiche, nel sistema signorile che controllava le risorse agricole e umane, e nelle relazioni commerciali e finanziarie con i paesi confinanti.

La presenza della Francia contribuiva ad approfondire la dialettica fra Torino e Chambéry, a partire dalla difesa militare del territorio, col problema irrisolto per tutta l'età moderna della difficoltà per lo stato sabaudo, se non l'impossibilità, di difendere la Savoia (al pari di Nizza) in ogni occasione in cui lo scontro avveniva con la monarchia d'oltralpe. Anzi la Savoia risultò a più riprese un'area da consegnare all'occupazione, per lo più inevitabile, al fine di servirsene come pedina di contrattazione internazionale.

Il grande cambiamento nella configurazione territoriale degli spazi politici avvenne all'inizio del Seicento quando per ottenere i territori del marchesato di Saluzzo, caduti in mano della Francia, Carlo Emanuele I cedette col trattato franco-sabaudo di Lione (1601) alcune province della Savoia (Bugey, Bresse, Gex e Valromey), per assicurarsi il marchesato di Saluzzo e le fortezze di Exilles e Bricherasio, smembrando così una parte della indifendibile Savoia, e soprattutto segnando come propria frontiera il Rodano, questo fiume così presente alla memoria degli storici (basti pensare al sistema Reno-Rodano celebrato da Huizinga ${ }^{2}$ per connettere la civiltà borgognona al Mediterraneo e alle ricerche di Lucien Febvre che ha saputo vedere il Reno come una strada liquida intorno al quale si confrontavano modelli di civiltà $)^{3}$.

Per difendere il nuovo assetto lo stato sabaudo avrebbe lasciato a lungo abbozzate le vie di comunicazione fra Piemonte e Savoia, e avrebbe spostato al di qua delle Alpi i gendarmi delle frontiere: le fortezze, i dazi, le truppe. Ma questa scelta favorì inevitabilmente l'identità di una regione di frontiera, come la Savoia, abituata a gravitare sulla Francia e, in caso di guerre, ad 
essere controllata dai Francesi, rafforzando tradizioni di autonomia legate a dimensioni locali, pur in un quadro di prevalente fedeltà dinastica ${ }^{4}$.

Non sono tanto le suddivisioni del potere politico ad essere percepite come qualificanti da chi, come i viaggiatori, si spostava da un territorio all'altro. Ricordiamo ad esempio che il modello interpretativo dello «spartiacque» lungo la cresta delle Alpi si era affermato già nel XV secolo, non tanto in opere topografiche, quanto piuttosto nei resoconti di viaggio, come nel caso dell'umanista fiorentino Leonardo Bruni. Per molti viaggiatori la catena delle Alpi appariva come una vasta zona di confine, come cesura culturale, come un mondo a sé stante tra l'Italia e l'Europa (fosse essa la Francia, la Svizzera o la Germania)5. Occorre piuttosto vedere la relazione TorinoChambéry come segmento di una più ampia dimensione territoriale, che si articola dalla Francia meridionale alla Svizzera, dal sud della Germania all'Italia, e che ingloba il bacino del Rodano, il lago di Ginevra e le Alpi occidentali e lambisce lo spazio mediterraneo-alpino fra Nizza, Francia e Piemonte. In tale ottica le comunicazioni culturali e politiche si comprendono con maggiore profondità in quanto sono sottratte al solo sistema dello stato e considerate in un quadro più largo di problemi. Stato e confini non racchiudono culture, lingue, tradizioni, esperienze.

In che misura si entrava in una cultura diversa passando le Alpi? Certo è che una delle esperienze più significative del viaggio, un indice quasi sicuro del fatto di entrare in una cultura differente dalla propria, varcando un confine culturale, è sicuramente il cambio della lingua. Al di là del problema generale per cui nella vecchia Europa i confini linguistici non coincidono praticamente mai con i confini politici, nella zona di cui ci stiamo occupando ci sono due difficoltà particolari nell'affrontare il concetto di confine linguistico. Da una parte sulle Alpi, come in molte regioni improntate da commercio e transito, numerosi gruppi umani sono in grado di farsi intendere in due o più lingue. Questo vale soprattutto per commercianti, carrettieri, artigiani e osti che operano lungo le vie di transito; ma vale anche per le élites funzionariali presenti nelle città. Dall'altra parte il bilinguismo è istituzionalizzato: negli stati sabaudi leggi e decreti di Sua Maestà sono redatti in italiano e francese e almeno fino al Settecento il francese è lingua usata a Torino dalle élites dello stato e di corte.

I viaggiatori si possono sommariamente collocare in due categorie ${ }^{6}$. Alla prima appartengono i viaggiatori abituali e culturalmente generici: colporteurs, ambulanti, mercanti, negozianti e fabbricanti, migranti temporanei,

4 Raviola 2007; Merlin/Rosso/Symcox/Ricuperati 1994; Carpanetto 2007.

5 Pastore 2007; Ciancio 2007.

6 Roche 2003. 
compagnons, persone che viaggiano per affari di famiglia o per salute, mercenari, rifugiati in tempi di disordini, e così via. La seconda accoglie $i$ viaggiatori colti, i viaggiatori-professionisti che seguono tipologie specifiche di mobilità, come i medici nel loro tour médical sapientemente indagato da Daniela Vaj attraverso il prisma dei ginevrini Louis Odier e Louis-André Gosse $^{7}$, e come i viaggiatori scientifici, che si spostano per visitare i luoghi della scienza, per analizzare la natura, per indagare gli spazi intatti e insoliti. Lungo tali itinerari l'uomo del Settecento perviene alla scoperta della montagna, che diventa un autentico laboratorio da porre sotto indagine interrogandone clima, altezza, particolarità botaniche e geologiche, caratteri chimici dell'aria e così via. Fondamentale l'apporto offerto dagli studi di botanica. A Torino, inaugurato nel 1729, il giardino dei semplici fu uno dei fiori all'occhiello della monarchia, illustrato dal magistero di Vitaliano Donati, botanico e viaggiatore scientifico, che il re inviò in Oriente, e soprattutto di Carlo Allioni, il Linneo piemontese. Dalla cultura botanica mosse i primi passi la scoperta, meglio l'invenzione della montagna, vissuta come laboratorio geologico e naturalistico, a cui seguiranno nell'Ottocento gli sguardi romanticamente fissati sul paesaggio inteso come riflesso dell'anima, e, come questa, in bilico tra l'orrido e il sublime. Non è un caso che la prima ascensione al Monte Bianco sia stata effettuata da un medico, laureatosi a Torino, Michel Gabriel Paccard, di Chamonix, cultore di botanica e mineralogia, che in compagnia del valdostano Jacques Balmat toccò la vetta l'8 agosto 1786, lasciando di stucco il ginevrino De Saussure, che si stava anch'egli preparando all'impresa $^{8}$.

\section{Daquin e il termalismo}

Uno dei principali scenari che dà vita allo scambio materiale e culturale tra Piemonte e Savoia è rappresentato dall'interesse scientifico, quale emerge a fine Settecento intorno ai temi della medicina, della botanica, dell'agricoltura, delle scienze naturali. Nasce allora un'inedita attenzione al controllo e all'utilizzo delle risorse ambientali, nella quale assumono un'importanza cruciale i temi del termalismo. Si tratta di un campo ancora poco esplorato in cui la ricchezza dei materiali è testimonianza essa stessa della rilevanza del tema9 ${ }^{9}$ Sono fonti che continuamente richiamano il nesso PiemonteSavoia e lo collocano in una prospettiva di dimensioni europee. Basti pen-

7 Vaj 2002.

8 Joutard 1986.

9 Taiani 1991. 
sare all'opera di ricognizione delle risorse termali promossa dall'Accademia delle Scienze di Torino nel 1784, che vide protagonista il medico Costanzo Bendetto Bonvicino, uno dei leaders della chimica piemontese tra tardo '700 ed età francese.

In Savoia, la figura di Joseph Daquin, autore del primo trattato completo pubblicato nel ' 700 sulle acque di Aix-les-Bains, domina la scena della nuova scienza. Daquin simboleggia efficacemente l'immagine del médecin-philosophe, nel quale la passione per la ricerca si associa allo spirito filantropico rivolto all'utilizzo delle competenze in opere di interesse pubblico. Spirito illuminato, uomo della repubblica delle lettere e al tempo stesso funzionario al servizio della sanità pubblica: diversi archetipi del nuovo savant si attagliano alla sua vita.

Nato a Chambéry nel $1732^{10}$ da una famiglia di funzionari della magistratura, Daquin si formò all'università di Torino, alla scuola di Ignazio Somis ${ }^{11}$, medico del re e protettore di quella pattuglia di medici, militari, nobili colti, che si stavano organizzando come gruppo accademico e dai quali sarebbe derivata l'Accademia delle Scienze. Decisivo fu l'incontro con il naturalista veneto e professore a Torino Vitaliano Donati ${ }^{12}$, ma ancor più il tour di specializzazione post lauream alle università di Montpellier e Parigi. Ritornato nel 1762 a Chambéry, Daquin fu nominato medico all'Hôtel-Dieu e nel 1788 agli Incurables, incarichi che terrà fino alla morte. Contemporaneo e amico di Rousseau, collezionista e bibliofilo, socio di numerose accademie, fu tra i fondatori della Société d'Agriculture di Chambéry ${ }^{13}$, nel 1772, di cui divenne segretario perpetuo. Come ha spiegato Jean Nicolas, tuttavia la Société stentò a decollare per effetto dei conflitti interni alla società savoiarda innescati dall'affrancamento dei feudi (la politica anti feudale operata dalla monarchia sabauda) e che videro una parte dei soci, favorevoli alla riforma e riuniti attorno a Daquin, scontrarsi col gruppo filo aristocratico che intendeva schierare la Société contro la riforma.

Fu solo durante la Rivoluzione che il ruolo di Daquin potè esprimersi in una vasta azione nel campo sanitario. Eletto nel dicembre 1792 alla municipalità di Chambéry con 343 suffragi, secondo nelle liste di preferenza, fu incaricato di tutte le questioni di polizia medica. Nominato professore di storia naturale all'Ecole Centrale du département du Mont-Blanc dalla sua fondazione, nel settembre 1797, fino al 1801, esercitò le funzioni di presidente del consiglio di amministrazione e di polizia dell'Ecole e quella di direttore

10 Guilland 1852; Bonino 1824-25, II, 474-476.

11 Vernazza 1784; Bonino 1824-25, II, 225-235.

12 Bonino 1824-25, II, 145-176; Grmek 1992, 62-64; Scalva 2001.

13 Grillet 1807, I, 194. Sulle vicissitudini della Société: Nicolas 1978. 
dell'Orto botanico, la cui creazione era già stata auspicata da Rousseau. Incaricato di effettuare, per conto del Dipartimento, le osservazioni meteorologiche, consegnò i risultati agli Annuaires du Mont-Blanc per gli anni XII, XIII e XIV, nei quali fece valere una lunga consuetudine che datava dal 1770, quando aveva cominciato a registrare regolarmente e quotidianamente i valori di pressione, temperatura, grado di umidità dell'aria e la forza dei venti, servendosi in particolare del Saggio meteorologico del padovano Giuseppe Toaldo, di cui curò la traduzione apparsa nel 1784, arricchita di note e commenti ${ }^{14}$. Il fulcro dei suoi lavori si espresse nella celebre Topographie médicale de la ville de Chambéry et de ses environs ${ }^{15}$, saggio di eziologia e sociologia medica, in cui l'autore seguiva «il modello francese secondo il quale i canoni del lavoro erudito sono sostituiti da una chiara denuncia dei disordini della vita sociale e da un sollecito invito agli amministratori affinché vi pongano rimedio» ${ }^{16}$. Preceduta dalla Lettre aux amateurs de l'agriculture (1771) e dal Mémoire sur la recherche des causes qui entretiennent les fièvres putrides à Chambéry $(1774)^{17}$, la topografia di Daquin si iscrive a pieno titolo in quella corrente di studi allora all'apice del successo, incentrata sul genere della «corografia georgico-iatrica». Frutto della filantropia dei lumi, queste «mappe del reale» si avvalevano degli strumenti della chimica, della fisica, della meteorologia, ed anche della storia, per delineare un universo totale fissato in un circoscritto spazio locale, fatto di presente e di passato, di peculiarità climatiche ed ecologiche, e percorso da patologie ricorrenti e tipologizzabili. L'indagine di Daquin non si limitava a svolgere un resoconto delle acque e delle arie, ma prendeva posizione su molti terreni della pratica sanitaria. A partire dalla denuncia della perdurante negligenza nel sottomettere i bambini alla vaiolizzazione, lo stesso impulso a quantificare l'andamento demografico aveva una finalità politica in quanto suggeriva interventi a difesa della salute. Daquin denunciava come piaga sociale gli abbandoni dei neonati ${ }^{18}$, propugnava misure di igiene da attuarsi con interventi edilizi e urbanistici, caldeggiava un rinnovo totale dei quattro nosocomi di Chambéry - Hôtel-Dieu, Charité, Incurables, Orphelins - e mostrava il volto sociale della malattia, intesa come portato delle differenze di ceto, indagando le patologie degli indigenti, dei mendicanti, dei lavoratori urbani ${ }^{19}$. Allargava il suo sguardo di medico riformatore dalla città alle campagne, dando spazio

14 Daquin 1784. Su Toaldo: Bozzolato 1984; Casati 1990,17-41.

15 Daquin 1787.

16 Montaldo 1998, 36.

17 Daquin 1771, 1774.

18 Daquin 1773, 250-251; Nicolas 1989, 41.

19 Daquin 1787, 107. Sulla medicina in Piemonte è fondamentale: Maffiodo 1996. Per l'università di Torino: Carpanetto 1998; 2002a; 2002b. Sul termalismo in Savoia: Francoeur 1826. 
ai problemi dell'agricoltura e discutendo le cause della crisi di produzione in cui versava l'economia savoiarda: scriteriati disboscamenti, errate rotazioni, mancanza di presidio veterinario, scambio ineguale e penalizzante con i paesi limitrofi, erano alcuni dei motivi ricorrenti di un'analisi ispirata dai canoni della fisiocrazia.

«La médecine ne s'est occupée jusqu'ici que de moyens physiques de traiter les fous; elle a négligé ceux qu'on pourrait tirer de la philosophie et certainement il y aurait beaucoup à dire sur ce point $»^{20}$ : questo passo della topografia preannunciava il nuovo oggetto di ricerca intrapreso dal Daquin. La pubblicazione della Philosophie de la folie ${ }^{21}$, avvenuta a Chambéry nel 1791, renderà note le sue idee sul trattamento dell'alienazione mentale. La malattia mentale poteva essere curata mediante un'intensa psicodinamica personale tra medico e paziente, accentuando i metodi cosiddetti «morali»: dolcezza, persuasione, prudenza, propositi consolanti dovranno essere, nella maggior parte dei casi, i soli soccorsi da impiegare ${ }^{22}$. Opera pionieristica che anticipava, come è stato rilevato, i temi del Traité di $\mathrm{Pine}^{23}$, frutto di un medico di provincia che sollevava «solitairement le voile d'un art nouveau» ${ }^{24}$, l'opera aprì un campo nuovo all'attenzione medica.

Esponente della massoneria, in quel periodo variamente connessa con il mondo scientifico, la sua firma figura, a partire dal 1765 , in qualità di primo sorvegliante nel regolamento della loggia dei Trois Mortiers (eretta nel maggio 1749 a Chambéry) che abbandonerà nel 1774 per unirsi ad un nuovo tempio a maggioranza aristocratica, quello della Sincérité, sorto da una scissione della precedente e in cui Daquin ricoprirà gli incarichi di guardasigilli, cancelliere, archivista, ricevendo al suo indirizzo personale la corrispondenza della $\log \operatorname{lia}^{25}$. La missione assegnata dalla Sincérité ai suoi adepti di occuparsi dei doveri dell'individuo relativamente alla società civile e alle grandi questioni della politica, si coniuga con le preoccupazioni di coloro che, come Daquin si adoperavano attivamente per il progresso materiale e morale.

Come detto, Daquin potè acquisire strumenti istituzionali dopo la conquista francese della Savoia, quando fu membro del Jury médical nel Dipartimento e segretario del comitato centrale per la vaccinazione in Savoia ${ }^{26}$. Il suo contributo alla profilassi jenneriana fu ribadito con la pubblicazione nel

20 Daquin 1787, 115.

21 Daquin 1791.

22 Rey 1996, 195-257.

23 Pinel 1801; Pinel 1797-98.

24 Guilland 1852,23. La seconda edizione della Philosophie de folie, pubblicata a Chambéry nel 1804, fu dedicata a Pinel.

25 Vermale 1912.

26 Carpanetto 2004. 
1801 della Lettre sur la vaccine ${ }^{27}$ e con la traduzione, all'età di 81 anni, del trattato sul vaccino del milanese Luigi Sacco, una delle opere di riferimento del movimento vaccinale europeo.

\section{Analisi chimica e applicazioni terapeutiche delle acque di Aix}

Nel Settecento grazie al crescente interesse per le cure naturali, il termalismo tornava a vivere una stagione particolarmente felice, sottolineata dal fatto che i luoghi delle acque richiamarono i riti della socialità nobiliare. Era la nobiltà, infatti, ad essere maggioritaria nel microcosmo termale del XVIII secolo e a riscoprire, attraverso le virtù delle acque, il loisir thermal, ossia l'occasione dell'incontro in quelli che erano diventati veri e propri santuari della salute fisica.

Un interesse del tutto nuovo verso le proprietà terapeutiche delle acque si concretizzò a partire dalla seconda metà del Settecento anche in ambito culturale, con un approccio medico-scientifico che soprattutto in Francia si precisò grazie alle iniziative dell'Académie des Sciences e della Société de médecine di Parigi. La prima promosse una serie di ricerche sulle sorgenti termali del regno, mentre la seconda raccolse una collezione di monografie locali, corredate dai rapporti annuali dei médecins-inspecteurs des eaux minerales. Nel campo della ricerca si moltiplicarono i manuali e i trattati di idrologia, con la pubblicazione dei lavori di Buchoz e di Carrère, che misero a disposizione sintesi globali sul termalismo francese ${ }^{28}$.

Anche in Italia il progresso della chimica e la volontà di favorire lo sfruttamento economico delle risorse termali contribuirono a diffondere il nuovo filone di letteratura chimico-naturalistica ${ }^{29}$. Scrissero su questi temi nomi importanti delle medicina italiana, tra i quali Anton Maria Lorgna con le Osservazioni fisiche intorno all'acqua marziale di Recoaro, stampate a Vicenza nel $1780^{30}$. Lazzaro Spallanzani, pur occupandosi primariamente della natura chimica dell'aria, effettuò esperimenti sulle acque di Scandiano nel corso del 1798, riuscendo ad isolarne i componenti gassosi ${ }^{31}$. Ai medesimi risultati era giunto Luigi Galvani, autore del saggio De aeriformibus principiis thermarum Porrectarum, letto all'Accademia delle Scienze di Bologna nel $1789^{32}$. Le Relazioni di alcuni viaggi fatti in diverse parti della Toscana, a

27 Daquin 1801.

28 Buchoz 1772-74; Carrère 1785.

29 Abbri 1989, 425.

30 Lorgna 1998.

31 Montalenti/Rossi 1982. Per le ricerche chimiche dell'acqua: Manzini 1990; Mazzarello 2004. 32 Abbri 1991, 101-111. 
cura di Giovanni Targioni Tozzetti, testimoniavano di terme famose ma anche di alcune ancora sconosciute, come le Caldane di Volterra, al cui fango, debitamente trattato, venivano attribuite proprietà terapeutiche nella cura delle dermatosi ${ }^{33}$. Di fondamentale importanza per il territorio e le fonti montecatinesi fu il trattato di Alessandro Bicchierai Dei Bagni di Montecatini, pubblicato nel 1788 dopo lunghe analisi, da cui emergono le principali linee di sviluppo dell'indagine sperimentale europea ${ }^{34}$. Infine, per il Regno di Napoli, va ricordato il ponderoso Trattato delle acque minerali in generale e in particolare di Nicola Andria, con un approfondimento sulla storia delle terme di Ischia. L'opera, data alle stampe nel 1775, venne riedita nel 1783 in due volumi ${ }^{35}$.

Sulla scorta di quanto da tempo avveniva in Italia e nel resto d'Europa, anche l'Accademia delle Scienze di Torino rivendicò la propria autorità in materia di indagine chimica sulle acque, un settore peraltro riconosciuto della massima rilevanza per la politica sanitaria. Nella seduta del 18 luglio 1784 il vicepresidente Carlo Ludovico Morozzo propose «per suggerimento del signor Presidente d'intraprendere l'analisi delle acque minerali degli stati di S.M. e di suo proprio pensiero dato in iscritto un progetto di far l'analisi di quelle de' pozzi di Torino» ${ }^{36}$. L'insieme di queste iniziative prese i caratteri di un'impresa collettiva volta a chiarire progressivamente i meccanismi di formazione delle acque minerali e delle loro componenti e a promuoverne un più ampio uso terapeutico ${ }^{37}$.

Fu la Savoia la prima regione culturale del Regno di Sardegna che attivò un rinnovato sguardo sulle risorse termali a partire dalle ricerche di Joseph Daquin, il cui interesse per l'idroterapia risaliva al 1773, anno in cui pubblicò l'Analyse des eaux thermales d'Aix en Savoie ${ }^{38}$, il primo trattato completo sulle acque termali di Aix apparso nel Settecento.

L'Analyse riproduce, ammodernandolo, lo schema di analisi codificato da Robert Boyle un secolo prima nelle Short memoirs for the natural experimental history of mineral waters (1684-85). Di Boyle condivide la scansione in tre fasi dell'analisi di un'acqua minerale: le caratteristiche fisiche esibite alla fonte, i tests chimici sull'acqua e sul residuo terroso ottenuto per evaporazione ed infine gli effetti terapeutici. Lo schema che, secondo Ferdinando Abbri, «aveva una lunga storia, rimase sostanzialmente immutato per

33 Barsanti/Becagli/Pasta 1996.

34 Una politica per le terme 1985.

35 Ravaglia 1928.

36 Accademia delle Scienze di Torino, Archivio storico, Verbali originali manoscritti della Classe di scienze fisiche e matematiche, cat. $3^{\circ}$, mazzo 15, 1783-1798, seduta del 18 luglio 1784.

37 Duhot/Fontan 1963; Auby 1994; Usages et représentations de l'eau 1986.

38 Daquin 1773. 
tutto il Settecento, anche se notevoli progressi furono compiuti nel campo dell'analisi chimica ${ }^{39}$. Era stato riprodotto nei lavori di Charles Le Roy, De aquarum mineraliumi ${ }^{40}$, e di Antoine-Grimoald Monnet ${ }^{41}$, entrambi maestri di Daquin alle Università di Montpellier e Parigi.

Nella prima parte dell'opera, Daquin propone una classificazione generale delle acque minerali, distinte, sulla base del loro grado di temperatura, in acque calde o termali (aquae medicatae calidae) e acque minerali fredde $\mathrm{o}$ acidule (aquae medicatae frigidae vel acidulae). Tra queste ultime, le più comuni, Daquin ricorda le acque di Amphion nella provincia del Chablais, molto rinomate, che con le loro virtù salutari attiravano numerosi stranieri. La ricerca degli elementi che producono il calore impegnava i fisici da lungo tempo: secondo Daquin è verosimile che le acque incontrino, nel loro percorso sotterraneo, sostanze dette piriti; queste, a loro volta, venendo a contatto con l'acqua si inumidiscono e formano efflorescenze che, surriscaldandosi e decomponendosi, cedono all'acqua un grado di calore proporzionato alla temperatura e alla quantità delle stesse piriti. La teoria della decomposizione delle piriti fu una delle più persuasive spiegazioni allora adottate dalla scienza, accanto ad altre ipotesi che trovavano riscontro nei fenomeni elettrici, nella combustione del carbon fossile, nell'ossidazione dei metalli che formano le terre e gli alcali. Restava ancora attiva l'ipotesi più arcaica che insisteva sulla teoria del fuoco centrale del globo terrestre ${ }^{42}$.

Alla parte più propriamente analitica del lavoro di Daquin è anteposto un paragrafo che funge da proemio storico sull'origine delle terme e della città di Aix, in cui l'autore utilizzava competenze erudite, di storia antica, di antiquaria e di epigrafia, per risalire alle origini preromane delle acque e percorrerne la storia fino ai suoi tempi. Ma l'obiettivo centrale era l'analisi chimica, costruita sulla considerazione di tre fenomeni fisici rimarcabili: l'abbondanza delle acque, il loro odore, del tutto simile a quello dell'epate di zolfo (foie de soufre), e la quantità dei vapori che, condensandosi sulle pareti degli antri dove sgorgano le sorgenti, formano una materia biancastra e molle, che a prima vista potrebbe sembrare zolfo sublimato ma che in realtà non possiede alcuna delle sue proprietà. La temperatura delle acque, che si mantiene pressochè costante in tutte le stagioni, è misurata in $36^{\circ}$ Réaumur, e il peso specifico inferiore di 2 libbre rispetto a quello dell'acqua comune. Sulla superficie del bacino dell'acqua solforosa si trovano fiocchi (flocons) di colore citrino, più abbondanti in inverno che, una volta disseccati, si ridu-

39 Una politica per le terme 1985, 225.

40 Le Roy 1758 e 1771.

41 Monnet 1779.

42 Despine 1834, 47-52. 
cono in polvere. Secondo le esperienze descritte nel Traité des eaux minérales dal Monnet (di cui Daquin era stato allievo a Parigi) ${ }^{43}$, la loro presenza dimostra che le acque contengono una quantità - imprecisata - di zolfo. Dalla combustione di questi fiocchi Daquin rileva che essi sono costituiti da una terra calcarea unita allo zolfo; dall'unione di questi due minerali deduce la presenza di un terzo composto, conosciuto come foie de soufre (solfuro nella successiva nomenclatura lavoisieriana), generalmente ritenuto un ottimo dissolvente. L'odore intenso dell'epate di zolfo diminuisce sensibilmente nel trasporto dell'acqua, anche a breve distanza dalla fonte ed in modo più marcato se la si lascia raffreddare. Attraverso il processo di distillazione Daquin registra la perdita consistente del principio solforoso. Nell'analisi tramite evaporazione, ponendo sul fuoco 6 libbre di acqua, si forma sulla superficie del liquido una pellicola terrosa; fatta disseccare e combinata con l'acido vitriolico si evapora nuovamente fino alla cristallizzazione ottenendo una terra di natura alcalina (carbonato di calcio). Di seguito sono riportati i risultati ottenuti con l'impiego di alcuni reagenti, quali la noce di galla e i tre acidi minerali, vitriolico, nitrico e marino, comunemente utilizzati per questo tipo di esperimenti. L'esame effettuato sulla materia pastosa e biancastra formata dai vapori condensati dimostra la presenza, nella acque di Aix, di selenite o chaux vitriolée (solfato di calcio).

Daquin è attento nell'enumerare le differenze che sussistono tra le due fonti di Aix, provando come non possano dipendere dall'allume: non esiste infatti alcuna traccia di questa sostanza. L'odore sulfureo dell'eau d'alun è meno intenso e, raffreddandosi, si disperde molto più velocemente rispetto a quello dell'eau de soufre. Quando l'acqua è calda il suo sapore è acerbo e terroso ma resta comunque piacevole: questo fatto è dovuto, secondo il Daquin, ad una maggiore concentrazione di selenite; anche i fiocchi che galleggiano sulla superficie del liquido sono meno abbondanti. La sorgente d'alun avrebbe oltre tutto una qualità singolare: quella di far riprendere vigore alle erbe appassite ed alle piante avvizzite. Dopo aver tentato, con vari accorgimenti, di isolare l'allume che, genericamente, si pensava fosse contenuto nelle acque di questa fonte, non avendone riscontrata alcuna traccia, Daquin nega la sua presenza, proponendo di conseguenza che la sorgente non venga più designata, impropriamente, come eau d'alun, ma come «eau moins sulfureuse ou de la source supérieure».

E' evidente, e Daquin lo afferma più volte nel corso dell'opera, la confluenza nell'Analyse des eaux thermales d'Aix delle conoscenze acquisite nel corso del soggiorno parigino. La filosofia chimica francese della seconda 
metà del Settecento si richiamava a Stahl e si parlava, a questo proposito, di stahlismo francese $e^{44}$; il termine non vuole designare una particolare corrente o scuola unitaria ma soltanto alcuni medici, filosofi naturali, farmacisti che dominarono il panorama scientifico in quel periodo ${ }^{45}$. Istituzionalmente, nella Francia dell'ancien régime, la chimica era monopolio esclusivo di queste categorie di savants interessate a sviluppare metodi adatti ad ottenere sostanze terapeuticamente efficienti e non pericolose. Era perciò una disciplina analitica che aveva ben pochi riferimenti e collegamenti con la matematica e «non godeva né di popolarità né di attenzione tra i fisici, la cui opera era arrivata ad un alto grado di complessità teorica, anche per questo le teorie stahliane venivano considerate false, contraddittorie ed oscure» ${ }^{46}$. Il riferimento principale si fissa su Guillaume François Rouelle ${ }^{47}$, dimostratore di chimica al Jardin du Roi, maestro di due generazioni di chimici (tra i quali anche Daquin). Per conciliare la teoria di Stahl (che ammetteva solo l'acqua e la terra come elementi chimici dei corpi) con la scoperta di Hales sulla combinabilità chimica dell'aria, Rouelle introdusse una spiegazione di tipo aristotelico riconoscendo la validità, come elementi dei corpi e strumenti di cambiamento, del fuoco, dell'aria, dell'acqua e della terra. Ed era proprio l'accettazione dei quattro prima di Aristotele l'elemento comune che contraddistinse i cosiddetti «stahliani francesi».

Daquin fu anche discepolo di Pierre Joseph Macquer ${ }^{48}$, medico e chimico di fama, il quale, pur accettando il flogisto di Stahl, configurava la chimica come una scienza fisica: solo l'utilizzazione dei risultati della fisica poteva consentirle di giungere ad un alto livello di perfezione quantitativa. La chimica francese (e continentale in genere) era sorta in stretta relazione a discipline come la farmaceutica, la medicina e la mineralogia, in cui il problema dell'analisi delle sostanze, al fine di stabilirne la composizione, risultava primario ed il fuoco era il metodo usato principalmente per questo tipo di esperimenti. Sorsero questioni molto complesse, ed in particolare quella inerente il rapporto tra il fuoco (elemento dei corpi e strumento di analisi) ed il flogisto. Per i chimici francesi la natura e le proprietà del flogisto avevano un valore assoluto, a differenza di quanto asserivano i filosofi naturali inglesi, che interpretavano il flogisto come elemento capace di effettuare determinati cambiamenti in circostanze date. Sarà Joseph Priestley nel 1774 a tentare di esaminare la natura del flogisto nei suoi rapporti col fuoco, con

44 Abbri 1978.

45 Gillispie 1980.

46 Abbri 1978, 22.

47 Laissus 1964, 287-341.

48 Macquer 1749-1751 e 1766. Cfr. Ferrone 1988, 94. 
la luce e con l'elettricità ${ }^{49}$, prima che Lavoisier con il concetto di fluido igneo o calorico, offrisse la soluzione che avrebbe consentito di trovare un terreno comune tra fisici e chimici.

\section{La terapia delle acque}

La seconda sezione del trattato di Daquin, Des différentes façons de prendre les eaux, dedicata alla applicazione terapeutica delle acque, muoveva da una descrizione delle parti solide e liquide del corpo umano che mostrava il suo tradizionale impianto galenico.

Il est constant et prouvé par l'expérience, que cette substance (foie de soufre) porte au moyen du véhicule aqueux, dans la masse des humeurs, les pénétrera jusques dans la plus petite de leurs molécules, divisera leur cohésion mutuelle, les rendra plus fluides et par conséquent plus aptes au mouvement; ainsi la masse humorale augmentera de volume parce qu'à chacune de ses particules, il s'en joindra une des eaux sulfureuses; leurs douces oscillations faciliteront encore la circulation, la rendront beaucoup plus égale et plus uniforme. ${ }^{50}$

Nell'indagare i risvolti terapeutici del termalismo un riferimento prioritario era costituito dalla concezione ippocratica della cura naturale, sostenuta agli inizi del Settecento da Friedrich Hoffmann. Nella teoria hoffmaniana l'acqua veniva ad assumere un ruolo basilare come anti-spasmodico, nel caso di blocchi del flusso degli umori, divenendo in tal modo un eccezionale evacuante ${ }^{51}$. Su questi presupposti Daquin raccomanda l'uso delle acque termali, da assumere per via o interna o esterna: nel primo caso si ingeriscono pure o unite con latte o sali neutri; nel caso di applicazioni esterne si utilizzano le docce e i bagni. La descrizione dei metodi di impiego delle acque è particolareggiata e ricca di osservazioni. Innanzitutto viene indicato come periodo più favorevole per la terapia termale quello compreso tra maggio ed agosto, quando le acque, non più mescolate alla neve, risultano maggiormente efficaci. L'assunzione per via interna tramite bevanda è consigliata al mattino e a digiuno; si incomincia con una libbra (di liquido) per aumentarne la quantità fino a quattro e oltre, il tutto per una durata tra i dodici e ventuno giorni, a seconda dei casi. Per trattenerne le virtù è essenziale che si bevano alla fonte poiché, secondo un'opinione comunemente diffusa, le acque durante il trasporto evaporano facilmente perdendo il loro principio attivo. $\mathrm{Si}$ assumono in bicchieri di vetro, ad intervalli di mezz'ora: trascorso un po' di tempo si potrà anche prendere un leggero pasto, conveniente al gusto e alla condizione dei pazienti.

49 Priestley $1775-1776$.

50 Daquin 1773, 44.

51 Benedicenti 1951. 
L'uso terapeutico dei bagni richiede invece maggiori cautele: vengono consigliati due bagni tiepidi al giorno della durata di un'ora o un'ora e mezza, mentre i bagni caldi si riducono a sette-otto minuti. Non dimentichiamo che Daquin fu tra i primi a quell'epoca a parlare dell'applicazione dei fanghi, fino ad allora scarsamente adoperati: «Les boues pourroient devenir de quelque utilité, étant appliquées en topique, comme on emploie celles d'Acqui en Piémont et celles des eaux de Bourbonne en France: c'est une expérience à tenter que jusqu'ici personne, que je sache n'a entreprise $»^{52}$. Ne indica l'impiego tramite fomenti sulle parti malate, avendo la precauzione di sostituirli o umettarli ogni qualvolta incomincino a seccarsi; potranno inoltre servire come rimedio preparatore alle docce. Queste ultime sono certamente il metodo curativo più efficace tra quelli impiegati ad Aix. La forza dell'acqua dovrà essere proporzionale alla durata della doccia ed entrambe alla natura e al grado della patologia: nella maggior parte dei casi è opportuno per Daquin effettuare da dodici a quindici docce, della durata media di otto-dieci minuti, preferibilmente al mattino e, se necessario, anche alla sera. Una volta terminata l'immersione il paziente sarà avvolto in un panno di lana in modo da permettere la traspirazione e l'assorbimento attraverso i pori della pelle. L'acqua impiegata per le docce è quella della sorgente de soufre in quanto possiede «une vertu incisive et fondante, par la quelle elle rende fluides ces humeurs épaissies et les remettent en voie de circuler, en pénétrant jusques dans le tissu le plus ferré des membranes aponévrotiques, qui est le siège le plus ordinaire des rhumatismes $»^{53}$.

Tuttavia perché le acque siano veramente efficaci occorre seguire le prescrizioni mediche circa il loro uso, che riguarda non solo la frequenza delle prestazioni, ma anche il genere di vita da condurre alle terme secondo un regime - preservativo, curativo e conservativo - da affiancare alla terapia vera e propria: le indicazioni riguardano principalmente l'alimentazione, il movimento e il riposo. Una fitta precettistica di carattere igienico-alimentare viene proposta in queste pagine, affinché si presti attenzione all'aria che si respira, alla dieta, al sonno, all'esercizio fisico e, non ultimo, al controllo delle passioni, il cui smodato esercizio è giudicato nocivo alla salute. Le indicazioni sulle abitudini alimentari dei pazienti riguardano i cibi solidi e liquidi: tra questi ultimi l'acqua è naturalmente la più opportuna al mantenimento della salute: «On a cherché pendant longtemps un remède universel, l'eau est la seule qui puisse mériter ce titre. Elle convient, dit Fréderic Hoffmann dans la Dissertation, parfaitement à toute forte de constitution et à toutes fortes d'âges et de temps» ${ }^{54}$.

52 Daquin 1773, 126-127.

53 Grmek 1996, 348.

54 Daquin 1773, 84. 
Sono proscritti liquori e spezie; proibite le carni di manzo, gli stufati, i ragù e i dolci, per l'elevato contenuto di grassi. Suggerisce cibi poco elaborati, verdure, frutta, pane e riso, consigliato «après la douche, surtout à ceux qui sont atteints de douleurs rhumatismales dans les articulations ${ }^{55}$. L'esercizio fisico è indissolubilmente legato alla terapia termale: passeggiare bevendo, camminare in aperta campagna, approfittare della vicinanza del lago di Bourget per andare in barca, «car le léger balancement qu'occasionne l'agitation de l'eau, joint à l'amusement de la pêche et au bon air qu'on y respire, ne laissera pas de faciliter le libre cours des liqueurs qui croupissent dans les petits vaisseaux ${ }^{56}$ : sono queste le indicazioni al riguardo. Si sofferma inoltre sui benefici effetti dell'equitazione, ritenuta una sorta di panacea: «Pendant une heure ou deux le matin, ceux qui seront à Aix pour des maux de poitrine, des affections vaporeuses et hypocondriaques et pour des obstructions, doivent surtout choisir l'exercice du cheval, parce qu'il ballotte davantage tous les viscères et qu'en accélèrent la circulation des humeurs, il désobstrue les petits vaisseaux ${ }^{57}$.

Nella terza ed ultima parte del saggio Daquin illustra quali patologie siano curabili con le acque termali, riportando per ognuna di esse le osservazioni relative ai diversi casi clinici. Per quanto riguarda l'uso esterno offre resoconti di venti guarigioni felicemente risolte: si tratta perlopiù di dolori reumatici, rigidità alle articolazioni degli arti inferiori, lussazioni e fratture ma anche paralisi ed emiplegie. Inoltre tenta di dimostrare l'inattendibilità di un pregiudizio all'epoca molto diffuso secondo il quale non si doveva in alcun modo bagnare la gotta, riportando due casi, entrambi localizzati nelle estremità inferiori, efficacemente curati con pediluvi ed immersioni in acqua termale.

Mentre l'impiego esterno si rivolge quasi esclusivamente ad infermità di tipo reumatico o, più in generale, dell'apparato motorio, l'uso interno tramite bibita ha dimostrato la sua validità curativa in una gamma più vasta di malattie: in modo particolare in diversi casi di disturbi allo stomaco, itterizia, nefropatie, affezioni della vescica urinaria, dolori di petto dovuti a tosse, asma e tubercolosi polmonare, ma anche in presenza di eruzioni cutanee e scabbia. In tutto dieci osservazioni che allargano lo spettro di intervento su alcune patologie che a volte si rivelavano persino difficili da diagnosticare; la cura termale non è presentata da Daquin come un palliativo che potrebbe attenuare momentaneamente i sintomi della malattia senza intervenire in modo diretto sulle cause scatenanti, ma come rimedio specifico e mirato alla completa guarigione.

55 Daquin 1773, 79.

56 Daquin $1773,89$.

57 Porter 1996, II, 348. 


\section{Conclusioni}

Le ricerche sul campo e la scelta professionale indirizzata a superare antiche regole e inveterate abitudini risultano le due chiavi di lettura dell'opera di Daquin, e spiegano la continuità tra l'attenzione al termalismo e gli studi sulla psichiatria.

La Philosophie de la folie, che pubblicò all'età di 61 anni, esprime le potenzialità e le difficoltà di un medico che agiva lontano dai centri della cultura e della politica. Ignorata forse volutamente da Pinel e oscurata dagli astri della medicina di Parigi, la Philosophie portò al suo autore una notorietà postuma, quando Daquin sarebbe tornato all'attenzione degli psichiatri, compiendo un lungo viaggio sotterraneo nella cultura europea, e quando gli storici riusciranno a spiegare anche quei passi apparentemente più lontani dalla cultura razionalista dell'epoca dei lumi, come l'adesione al «lunatismo» (l'ipotesi di una relazione tra cicli della luna e malattia mentale), leggendoli alla luce del naturalismo sperimentale, del climatismo di Giuseppe Toaldo, delle correnti vitalistiche così vivaci nel secolo dei Lumi. Quella circolazione di idee che sembrava inizialmente circoscritta alla regione alpina e allo stato sabaudo, troverà nell'800 una dimensione allargata e il nome di Daquin verrà associato alla riforma terapeutica e alla stagione pionieristica della psichiatria, sottraendolo all'icona angusta dell'eroe locale.

\section{Bibliografia}

Abbri, Ferdinando, «De utilitate chemiae in oeconomia rei publicae. La rivoluzione chimica nel Piemonte dell'antico regime», Studi storici 2 (1989) 401-433

- «Chemistry turned upside down: aspects of the Italian debate on Lavoisier's theory», in: Ferdinando Abbri/Franco Crispini (eds), Atti del III convegno nazionale di storia e fondamenti della chimica (Cosenza 1991) 101-111

- La chimica del Settecento (Torino 1978)

Auby, Jean-François, Les eaux minéraux (Paris 1994)

Barsanti Giulio/Vieri Becagli/Renato Pasta (eds), La politica della scienza. Toscana e stati italiani nel tardo settecento (Firenze 1996)

Benedicenti, Alberico, Malati, medici e farmacisti. Storia dei rimedi traverso i secoli e delle teorie che ne spiegano l'azione sull'organismo (Milano 1951)

Bonino, Giovanni Giacomo, Biografia medica piemontese (Torino 1824-25, rp. Bologna 1987)

Bozzolato, Giampiero, Giuseppe Toaldo, Uno scienziato europeo nel Settecento veneto (Brugine 1984)

Buchoz, Pierre-Joseph, Dictionnaires minéralogique et hydrologique de la France (Paris 1772-74)

Carpanetto, Dino, «L'università nel XVIII secolo», in: Giuseppe Ricuperati (ed.), Storia di Torino, V, Dalla città razionale alla crisi dello Stato d'Antico Regime (1730-1798) (Torino 2002a) 187-232

- «L'università ristabilita», in: Giuseppe Ricuperati (ed.), Storia di Torino, IV, La città fra crisi e ripresa (Torino 2002b) 1065-1092

- Scienza e arte del guarire. Cultura, formazione universitaria e professioni mediche a Torino tra Sei e Settecento (Torino 1998) 
- Il pregiudizio sconfitto. La vaccinazione in Piemonte nell'età francese 1800-1814 (Pinerolo 2004)

- Torino e gli stati sabaudi (Milano 2007)

Carrère, Joseph/François Barthelemy, Catalogue raisonne des ouvrages qui ont été publiés sur les eaux minérales en général et sur celles de la France en particulier, avec une notice de toutes les eaux minérales de ce royaume (Paris 1785)

Casati, Stefano, «Giuseppe Toaldo: la luna, il saros e le meteore», Nuncius 5 (1990) 17-41

Ciancio, Luca, «Nuove prospettive alpine: qualche osservazione di metodo», Società e storia 117 (2007)

Daquin, Joseph, Lettre aux amateurs de l'agriculture (Chambéry 1771)

- Analyse des eaux thermales d'Aix en Savoie, dans laquelle on exposa les diverses manières d'user ces eaux, la méthode et le régime de vivre qu'il convient de suivre pendant leur usage et les différentes maladies pour lesquelles elles sont employées, avec plusieurs observations qui y sont relatives pour en constater les propriétés (Chambéry 1773)

- Mémoire sur la recherche des causes qui entretiennent les fièvres putrides à Chambéry (Chambéry 1774)

- Essai météorologique sur la véritable influence des astres, des saisons et changement de tems, fondé sur de longues observations et appliqué aux usages de l'agriculture, de la médecine, de la navigation, par Joseph Toaldo Vicentin, traduit de l'italien en français avec des notes du traducteur (Chambéry 1784)

- Topographie médicale de la ville de Chambéry et de ses environs (Chambéry 1787)

- La philosophie de la folie, où l'on prouve que cette maladie doit plutôt être traitée par les secours moraux que par les secours physiques; et que ceux qui en sont atteints éprouvent d'une manière non équivoque l'influence de la lune (Chambéry 1791)

- Lettre sur la vaccine (Chambéry an IX-1801)

Despine, Charles, Manuel de l'étranger aux eaux d'Aix en Savoie (Annecy 1834)

Donati, Vitaliano, Viaggio mineralogico nelle Alpi occidentali: Valle di Susa, Maurienne, Tarentaise, Valle d'Aosta e Faucigny, nell'estate 1751, curato da Giuse Scalva (Bologna 2001)

Duhot, Emile/Michel Fontan, Le thermalisme (Paris 1963)

Febvre, Lucien, Il Reno. Storia, miti realtà (Roma 1999, ${ }^{1} 1931$ )

Ferrone, Vincenzo, La nuova Atlantide e i lumi (Torino 1988)

Francoeur, Louis Benjamin, Notice sur la ville d'Aix en Savoie et sur ses eaux thermales (Chambéry 1826)

Gillispie, Charles Coulston, Scienza e potere in Francia alla fine dell'ancien régime (Bologna 1983, $\left.{ }^{1} 1980\right)$

Grillet, Jean Louis, Dictionnaire historique, littéraire et statistique des départements du MontBlanc et du Léman (Chambéry 1807, rp. Marseille 1973)

Grmek, Mirko D., «Vitaliano Donati», in: Dizionario biografico degli italiani (Roma 1992)

- (ed.), Storia del pensiero medico occidentale, II, Dal Rinascimento all'inizio dell'Ottocento (Roma/Bari 1996)

Guilland, Louis, Notice biographique sur le médecin Daquin (Chambéry 1852)

Huizinga, Johan, Herfsttijd der Middeleeuwen (Haarlem 1919); I ed. italiana, L'autunno del Medioevo (Firenze 1944)

Joutard, Philippe, L'invention du Mont Blanc (Paris 1986)

Laissus, Yves, «Le Jardin du Roi», in: Roger Taton (ed.), Enseignement et diffusion des sciences en France au XVIII siècle (Paris 1964) 287-341

Le Roy, Charles, De aquarum mineralium natura et usu propositiones (Monspelii 1758)

- Mélanges de physique, de chimie et de médecine (Paris 1771)

Lorgna, Anton M., Scienziato ed accademico del XVIII secolo tra conservazione e novità (Roma/Verona 1998)

Macquer, Pierre Joseph, Eléments de chymie théorique et pratique (Paris 1749-1751)

- Dictionnaire de Chymie (Paris 1766)

Maffiodo, Barbara, I borghesi taumaturghi. Medici, cultura scientifica e società in Piemonte fra crisi dell'antico regime ed età napoleonica (Firenze 1996)

Manzini, Paola, Il filosofo nel pozzo. La terra di Lazzaro Spallanzani tra storia e scienza (Firenze 1990) 
Mazzarello, Paolo, Costantinopoli 1786: la Congiura e la beffa. L'intrigo Spallanzani (Torino 2004)

Merlin, Pier Paolo/Claudio Rosso/Geoffrey Symcox/Giuseppe Ricuperati,Il Piemonte sabaudo. Stato e territori in età moderna (Torino 1994)

Monnet, Antoine-Grimoald, Traité des eaux minérales avec plusieurs mémoires de chimie relatifs à cet objet (Paris 1768)

- Nouvelle hydrologie (Paris 1772)

- Nouveau système de minéralogie, ou Essai d'une nouvelle exposition du règne minéral, auquel on a joint un supplément au «Traité de la dissolution des métaux», avec des observations relatives au «Dictionnaire de chimi» [de M. Macquer] (Bouillon 1779)

Montaldo, Silvano, Medici e società. Bartolomeo Sella nel Piemonte dell'Ottocento (Torino 1998)

Montalenti, Giuseppe/Paolo Rossi (eds), Lazzaro Spallanzani e la biologia del Settecento. Teorie, esperimenti, istituzioni scientifiche (Firenze 1982)

Nicolas, Jean, La Savoie au 18 siècle. Noblesse et bourgeoisie (Paris 1978) 2 vol.

- La révolution française dans les Alpes. Dauphiné et Savoie 1789-1799 (Toulouse 1989)

Pastore, Alessandro, «Nuove prospettive alpine: montagna, scienza e società», Società e storia 117 (2007)

Pinel, Philippe, Nosographie philosophique, ou la méthode de l'analyse appliquée à la médecine (Paris, an VI-1797-98)

- Traité médico-philosophique sur l'aliénation mentale, ou la manie (Paris an IX-1801)

Porter, Roy, «Strategie terapeutiche», in: Mirko D. Grmek (ed.), Storia del pensiero medico occidentale, II, Dal Rinascimento all'inizio dell'Ottocento (Roma/Bari 1996)

Priestley, Joseph, Experiments and observations on different kinds of air (London 1775-1776)

Ravaglia, Giuseppe, Bibliografia idrologica italiana (Roma 1928)

Raviola, Blythe Alice (ed.), Il problema della frontiera e lo spazio sabaudo (Milano/Angeli 2007)

Rey, Roselyne, «L'anima, il corpo e il vivente», in: Mirko D. Grmek (ed.), Storia del pensiero medico occidentale, II, Dal Rinascimento all'inizio dell'Ottocento (Roma/Bari 1996) 195-257

Roche, Daniel, «Circolazione delle idee, mobilità delle persone: continuità e rotture», in: Le radici storiche dell'Europa. L'età moderna, curato da Maria Antonietta Visceglia (Roma 2007) 127-140

- Humeurs vagabondes. De la circulation des hommes et de l'utilité des voyages (Paris 2003)

Taiani, Rodolfo, «L'acqua e la sua anima: il contributo della scienza chimica allo sfruttamento delle fonti di acqua minerale nella prima metà del XIX secolo», Nuncius 2 (1991) 83-107

Una politica per le terme. Montecatini e la Val di Nievole nelle riforme di Pietro Leopoldo (Siena 1985)

Usages et représentations de l'eau. Actes du III congrès national des sociétés savantes (Poitiers/ Paris 1986)

Vaj, Daniela, Médecins voyageurs. Théorie et pratique du voyage médical au début du XIX siècle (Genève 2002)

Vermale, François, La Franc-maçonnerie savoisienne à l'époque révolutionnaire d'après ses registres secrets, préface d'Albert Mathiez (Paris 1912)

Vernazza, Giuseppe, Elogio del conte Ignazio Somis di Chiavrie (Torino 1784) 